\title{
The Modern Trend of Cancer Treatment
}

$\mathrm{O}^{\mathrm{F}}$ the special reports issued by the Medical Research Council, so far as we are aware, only one has assumed an annual character. We refer to the reports on the "Medical Uses of Radium", which have been issued yearly from 1922. Taken together, they form a valuable, interesting and concise summary of the progress of radium therapy in Great Britain, and show the important part which has been taken by the Council in its development. Although primarily intended for workers in a particular branch of therapy, they may, nevertheless, be consulted with profit and interest by all who are interested in the treatment of malignant disease.

The latest of these reports, issued in 1935*, differs from its predecessors in that the space allotted to purely experimental work is approximately equal to that occupied by clinical results and details of treatment. In previous issues, clinical methods and their comparative values formed by far the greater part of the report; now, owing to standardisation of certain methods of therapeutic technique, less space is necessary for their description. By standardisation of methods it is not meant that the radiation treatment of malignant disease can be carried out simply as a routine; each case must be treated as a separate problem, and the details of treatment modified to meet individual requirements. It does, however, mean that substantial progress has been made in advancing from the empirical to the scientific treatment of cancer. Not many years ago, surgical operation alone was regarded as the only possible method of giving relief ; operations became more and more extensive in the hope of eradicating the disease, and, as is but too well known, were often ultimately ineffective. How complete has been the change in medical opinion during recent years can be seen from the figures quoted in the latest report, which are based upon the returns made by the various centres acting in collaboration with the Council. Since these centres include most of the great hospitals both in the metropolis and in the provinces, their practice may fairly be taken as representative of the best medical opinion of the day. Out of a total of 2,475 cases of malignant disease treated during the year 1934, only 612 , or 24.7 per cent, were treated by purely surgical methods; in the remaining 1,863 cases some form of radiation therapy was employed, with or without more * Medical Research Council. Special Report Series, No. 204 :
Medical Uses of Radium; Summary of Reports from Research Centres for 1934. Pp. $45+2$ plates. (London: H.M. Stationery Office.) 18. net. purely surgical methods; and in no less than 1,145 cases treatment was by radium alone, and in 251 by X-rays alone.

The most striking results of radiation treatment have been seen in cancer of the tongue and cancer of the uterus; in nearly all such cases now the treatment of choice is by radium combined with high-voltage X-rays. The treatment of cancer in other sites presents many technical and inherent difficulties, sometimes from inaccessibility of the primary growth or of the paths by which the disease may spread, and sometimes from a combination of both these adverse factors. Such cases are receiving close attention, and the effects of combined radiation and electro-surgery and of massive radium radiation by one- to five-gram units* are under investigation at various centres.

The story of the part taken by the Medical Research Council in the development of radium treatment in Great Britain may be worthy of a brief note. In the year 1919 it acquired from the Ministry of Munitions about $5 \mathrm{gm}$. of hydrated radium bromide, equivalent to about $2.5 \mathrm{gm}$. of radium element. In the first instance this was put in the charge of the Middlesex Hospital, and used for experimental work upon the action of radiation upon normal and malignant tissues. The report upon this work was issued in 1922, and forms the first volume of the series which has been continued to the present time. The radium was then divided between various centres, after having been suitably mounted at the Medical Research Council's expense. Throughout, the Medical Research Council has worked in the closest and most harmonious co-operation with the National Radium Commission, and the result has been the acquisition of a vast amount of valuable information.

We have said that in the latest report nearly half the space is devoted to experimental work; this work has been of a most varied character, ranging from experimental findings in pure physies to facts relating to the reactions of the tissues under irradiation. We sincerely hope that these purely experimental findings will continue to appear in the same volume along with the results of radiation therapy. Such a combination will serve to emphasise the essential need for the co-ordination and co-operation of physicists, biologists, chemists and other special workers with the clinicians who translate scientific findings into their practical applications for the treatment of disease.

* This is the so-called 'bomb' treatment, an absurd terminology which should be banished from scientific literature. 